\title{
The Efficacy of Intravenous versus Subcutaneous Recombinant Erythropoietin in Obese African-African Patients in a Southeast U.S. Dialysis Cohort
}

\author{
Éva Csongrádi ${ }^{1,2}$, Michael Shoemaker-Moyle ${ }^{1}$, Lajos Zsom ${ }^{1}$, \\ Catherine Wells ${ }^{1}$, Zsolt Lengvárszky ${ }^{3}$, Mihály Tapolyai ${ }^{4}$ \\ and Tibor Fülöp ${ }^{1^{*}}$ \\ ${ }^{1}$ Department of Medicine, University of Mississippi Medical Center, Jackson, MS, USA. \\ ${ }^{2}$ Department of Medicine, Medical and Health Science Centre University of Debrecen, \\ Hungary. \\ ${ }^{3}$ Department of Mathematics, Louisiana State University Shreveport, Shreveport, LA, USA. \\ ${ }^{4}$ Fresenius Medical Care, Semmelweis University, Budapest, Hungary.
}

Authors' contributions

This work was carried out in collaboration between all authors. Authors ÉC and TF designed the study and wrote the first draft of the paper. Authors MSM and LZ initiated he study and obtained initial Institutional Review Board approval for the study. Authors MSM and CW collected the data. Author ZL provided statistical advice and revised the first draft of the paper. Authors TF, MSM and ÉC performed most of the literature searches. Authors MT and $L Z$ provided critical intellectual input into the interval revisions of the paper. All authors read and approved the final manuscript.

Research Article

Received $2^{\text {nd }}$ August 2013

Accepted $21^{\text {st }}$ August 2013

Published $14^{\text {th }}$ September 2013

\section{ABSTRACT}

Aims: To correct renal anemia, subcutaneous (SC) route of recombinant human erythropoietin (rhuEPO) administration has been associated with increased efficacy and decreased dose requirements, when compared with intravenous (IV) route. The effect of obesity as a potential modifier during rhuEPO administration has not been well explored.

Study Design: Single-center, Longitudinal Cohort Study.

Place and Duration of Study: University of Mississippi Medical Center Outpatient Dialysis Unit, between February and November of 2009. 
Methodology: We performed IV to SC rhuEPO conversion for 86 in-center dialysis patients and, following a six-month equilibration period, we monitored outcomes over a period of three months. We obtained baseline demographic parameters, calculated Body Mass Index (BMI) and monitored iron saturation, ferritin, hemoglobin ( $\mathrm{Hgb})$ along with rhuEPO requirements. Patients were divided into 3 categories based on BMI [ $<25(n=27)$, $\left.25-35(n=38),>35(n=21) \mathrm{kg} / \mathrm{m}^{2}\right]$. Results are reported either as percents, means with SD or median with $25-75 \%$ interquartile range, as appropriate.

Results: The cohort was all African-American, 48.8\% male, aged 54.7 (13.3) years and BMI calculated at $29.9(7.4) \mathrm{kg} / \mathrm{m}^{2}$. Baseline iron saturation was $24(10.6) \%$, ferritin measured $641(277) \mathrm{ng} / \mathrm{mL}$. Hgb remained unchanged during the observation period: 11.1 (1.3) vs. $11.2(1.3) \mathrm{gm} / \mathrm{dL}$. Initial rhuEPO weekly dose for the entire cohort was $19,729(17,448)$ Units/week (U/week); final dose 17,482 (14,860) U/week, with close correlation between initial and final doses ( $r$ : $0.653, P<0.0001)$. Weekly rhuEPO dose remained virtually unchanged in BMI categories 1 and $2[13,927(10,938)$ vs. 13,297 $(10,247)$ U/week; $20,684(15,788)$ vs. 20,997 (17.917)] ( $P=$ NS for both) but decreased in the category 3: $25,459(24,403)$ vs. $16,444(12,749)(P=0.081)$. However, BMI had no independent effect in linear regression modeling with multiple covariates (age, BMI, iron saturation, ferritin) included.

Conclusion: Obesity may affect relative efficacy of rhuEPO conversion; additional studies may be needed.

Keywords: African-American; body mass index; end-stage renal disease; obesity; renal anemia; recombinant erythropoietin.

\section{ABBREVIATIONS}

$B M I=$ Body Mass Index; $C M S=$ Centers for Medicare and Medicaid Services; DOPPS = Dialysis Outcomes and Practice Patterns Study; ESRD = end-stage renal disease; IQR = interquartile range; IS = iron saturation (percent); IV = intravenous (route); $K D O Q I=$ Kidney Disease Outcomes Quality Initiative; rhuEPO = recombinant human erythropoietin; SC = subcutaneous (route); $S D=$ Standard Deviation.

\section{INTRODUCTION}

Successful management of chronic renal insufficiency involves addressing multiple metabolic abnormalities, including correction of renal anemia and relative erythropoietin deficiency with recombinant human erythropoietin (rhuEPO) administration [1,2]. The most common route of rhuEPO administration in United States is the intravenous (IV) route, in keeping with KDOQI guidelines favoring IV administration for patient convenience ${ }^{1}$. However, IV conversion has been associated with increased rhuEPO and intravenous iron requirements [3] and up to $30 \%$ less total dosing of rhuEPO might be required to achieve identical hemoglobin $(\mathrm{Hgb})$ goals via subcutaneous $(\mathrm{SC})$ route $[4,5]$. One theoretical criticism of SC rhuEPO dosing is the reduced or variable efficacy in obese patients due to decreased bioavailability. The data in obese women with regard to similarly sized hormones (follicle stimulating hormone, human chorionic gonadotropin) are conflicting [6,7]. Concerns regarding worsening BP control with rhuEPO administration, especially via IV route, persist in the medical literature $[8,9]$. Further, recombinant erythropoietin or similar analogs are expensive and achieved hemoglobin elevations in excess of $>12 \mathrm{gm} / \mathrm{dL}$ appeared harmful in large trials [10-12], as well as during meta-analysis of existing literature [13]. The recent 
change of U.S. Center for Medicare and Medicaid Services (CMS) reimbursement arrangement may have removed the incentives for administering excessive doses of rhuEPO for financial gains in dialysis units of the U.S. These rules eliminated the separate payment for injectables and created a "bundled" payment for all services related to endstage renal disease (ESRD) into a single payment entity. Our study aimed to answer the question whether Body Mass Index (BMI) could potentially influence the reduction of rhuEPO dosing during conversion from the IV to the SC route. Our theory was that such dose reduction will vary between defined BMl categories.

\section{MATERIALS AND METHODS}

\subsection{Study Population}

The University of Mississippi Medical Center (UMMC) provides care for a large $(\geq 100)$ cohort of predominantly African American dialysis patients at the Jackson Medical Mall Outpatient Dialysis Unit, a metropolitan in-center unit in Jackson, MS. After revising our anemia management protocol in March of 2009, we attempted to switch our entire patient population from IV to SC rhuEPO utilization. Patients were included in the study if they received rhuEPO for management of ESRD-associated anemia and switched from IV to SC dosing during March of 2009. Additional enrollment criteria were African American race and a dialysis vintage of $\geq 3$ months. Of all the qualifying 90 subjects, four refused to undergo the SC conversion, leaving us with 86 patients. We undertook a retrospective chart review study of all patients $(\mathrm{N}=86)$ undergoing such conversion, after monthly laboratory tests have been obtained and subsequently maintained on such regimen at least until December of 2009, when the examination period ended. We collected data both at baseline (February and March, 2009) and, after a six-month equilibration period, during a follow-up period (September, October, November of 2009). We collected data on both demographic and anthropometric (age, gender, race, weight, body length and calculated Body Mass Index) as well as laboratory variables (hemoglobin, hematocrit, iron saturation, ferritin and reticulocyte count). Doses and route of rhuEPO were recorded both at baseline and during the follow-up period. Anemia management was executed according to our institutional dialysis-associated anemia management protocol, which could be overruled only by the patients' physicians. The anemia management protocol included an IV iron protocol and most of the patients received iron sucrose at a dose 50-100 mg weekly. The study was reviewed and approved by the University of Mississippi Human Research Office (formerly: Institutional Review Board) and approved in accordance with the revised 1983 Helsinki Declaration (IRB approval number \#2010-0028).

\subsection{Measurements}

Age was the number of full calendar years completed since birth. Gender was self-reported and either male or female. Ethnicity was exclusively African-American and self-defined. Weight was recorded as the physician-prescribed ideal ("target") weight for the recoded month. BMI was calculated based on patients' height and weight, and expressed as $\mathrm{kg} / \mathrm{m}^{2}$. Laboratory test results were provided via contracted third-party laboratory services provider (Spectra Laboratories, Inc.; Milpitas, CA). Medical records were updated within the month of data collection. 


\subsection{Statistical Methods}

Data on patient characteristics are shown as mean \pm standard deviation (SD) for continuous variables or percentages for categorical variables. Select variables (rhuEPO/week, rhuEPO/week $/ \mathrm{kg}$ of body weights) were also reported with medians and $25-75 \%$ interquartile range (IQR). Partly to minimize month-to-month variations and to decrease the number of missed observations, the results from the months before the conversion (February/March) summed up and averaged together for data analytic purposes. Similarly, the observations from the outcome period (September/October/November) were analyzed together. Patients were divided into three categories based on their BMI $\left(<25,25-35\right.$, and $\left.\geq 35 \mathrm{~kg} / \mathrm{m}^{2}\right)$. Paired samples T-test and linear regressions were utilized for data analyses. Statistical analysis was performed using SPSS 18 (IBM Corporation, Armonck, NY).

\section{RESULTS AND DISCUSSION}

Baseline characteristics of the cohort are presented in Table 1. At the start of the study, all patients were exclusively on IV rhuEPO (Epoetin alfa, Amgen Inc., Thousand Oaks, CA); molecular weight: 30,400 Daltons. The study cohort was an elderly middle-aged, exclusively African-American cohort, gender balanced and generally overweight (mean BMI $29.9 \pm 7.4)$. According to BMI categories: $27(31.3 \%)$ patients had $\mathrm{BMI}<25,38(44.1 \%) \mathrm{BMI}$ of $25-35$, and $21(24.4 \%)$ had $\mathrm{BMl}>35 \mathrm{~kg} / \mathrm{m}^{2}$. Monitored parameters did not meaningfully change during the February to March period, with the exception of ferritin $(P=0.045)$. The IV to SC conversion took effect during or shortly after the monthly laboratory studies have been obtained in March. During the six months equilibration period, eleven patients had been lost for follow-up due to death, renal transplantation, transfer to another units or conversion to peritoneal dialysis. At the end of the observation period, data from $72(83.7 \%)$ patients were available who successfully converted and maintained on SC rhuEPO. A consistent and otherwise unchanged anemia management protocol was used for both periods of observation. The cohort's rhuEPO utilization during this period is shown in Fig. 1. We observed a remarkable stability of hemoglobin, iron saturation and ferritin during this period ( $P=\mathrm{NS}$ for change). Recombinant erythropoietin use of the cohort, separated according to $\mathrm{BMI}$, during summarized baseline and follow-up period is shown in Table 2. Initial rhuEPO weekly dose for the entire cohort was 19,729 $(17,448)$ Units/week (U/week); final dose $17,482(14,860) \mathrm{U} /$ week, with close correlation between initial and final doses ( $r$ 0.653, $P<0.0001$ ). Comparing the mean weekly IV rhuEPO dosing for baseline (February/March) with SC dosing during the follow-up period (September-October-November), there was a small decrease in rhuEPO utilization for the entire cohort: 2,247 Units/week or $-11.4 \%$, which was not significant $(P=0.205)$. Separated according to BMI categories, in category 1 (lean) we observed minor, non-significant reduction (630 Units/week; $-4.5 \%)$ of rhuEPO use, whereas in category 2 (overweight/class 1 obese) a trivial rise (313 Units/week) during conversion, both of these changes not being statistically significant. However, in category 3 $(\mathrm{BMI} \geq 35)$ we noted a large decrease of 9,017 Units/week (35.4\%), showing a strong trend, albeit not reaching statistical significance $(p=0.081)$. However, BMI had no independent effect in linear regression modeling with multiple covariates (age, BMI; age, BMI, iron saturation, ferritin) included $(P=\mathrm{NS})$. 
Table 1. Cohort characteristics

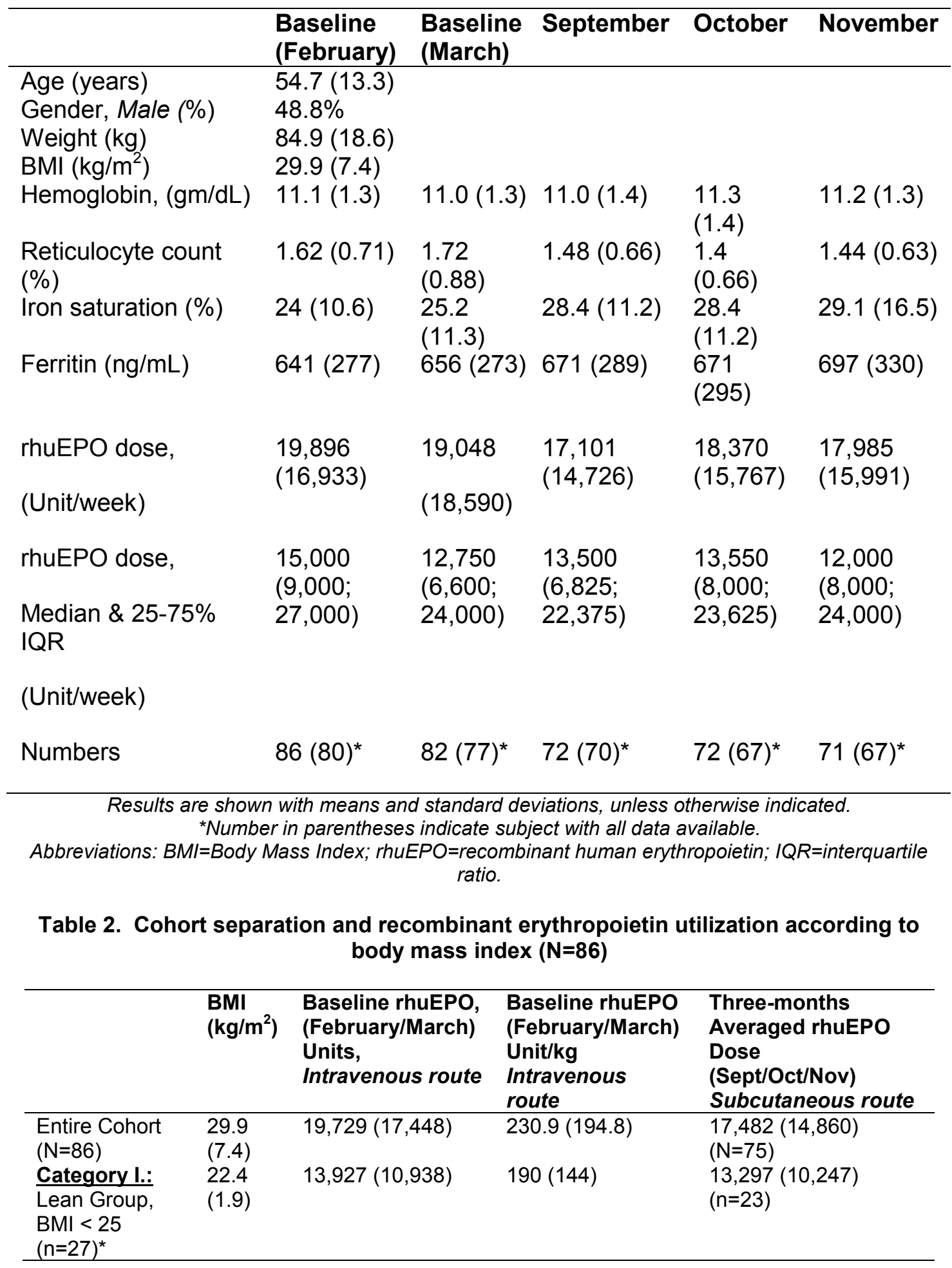




\begin{tabular}{|c|c|c|c|c|}
\hline $\begin{array}{l}\text { Category Il.: } \\
\text { Normal weight } \\
\text { - Class I } \\
\text { obesity Group, } \\
\text { BMI 25-35 } \\
(n=38)^{*}\end{array}$ & $\begin{array}{l}29.3 \\
(2.8)\end{array}$ & $20,684(15,788)$ & $219.3(173.1)$ & $\begin{array}{l}20,997(17,917) \\
(n=33)\end{array}$ \\
\hline $\begin{array}{l}\text { Category Ill.: } \\
\text { Class } 2 \text { Obese, } \\
\text { BMI > } 35 \\
(n=21)^{*}\end{array}$ & $\begin{array}{l}40.5 \\
(4.5)\end{array}$ & $25,459(24,403)$ & $250.4(259.3)$ & $\begin{array}{l}16,442(12,749) \\
(n=19)\end{array}$ \\
\hline
\end{tabular}

Abbreviations: $B M I=B o d y$ Mass Index; rhuEPO=recombinant human erythropoietin.

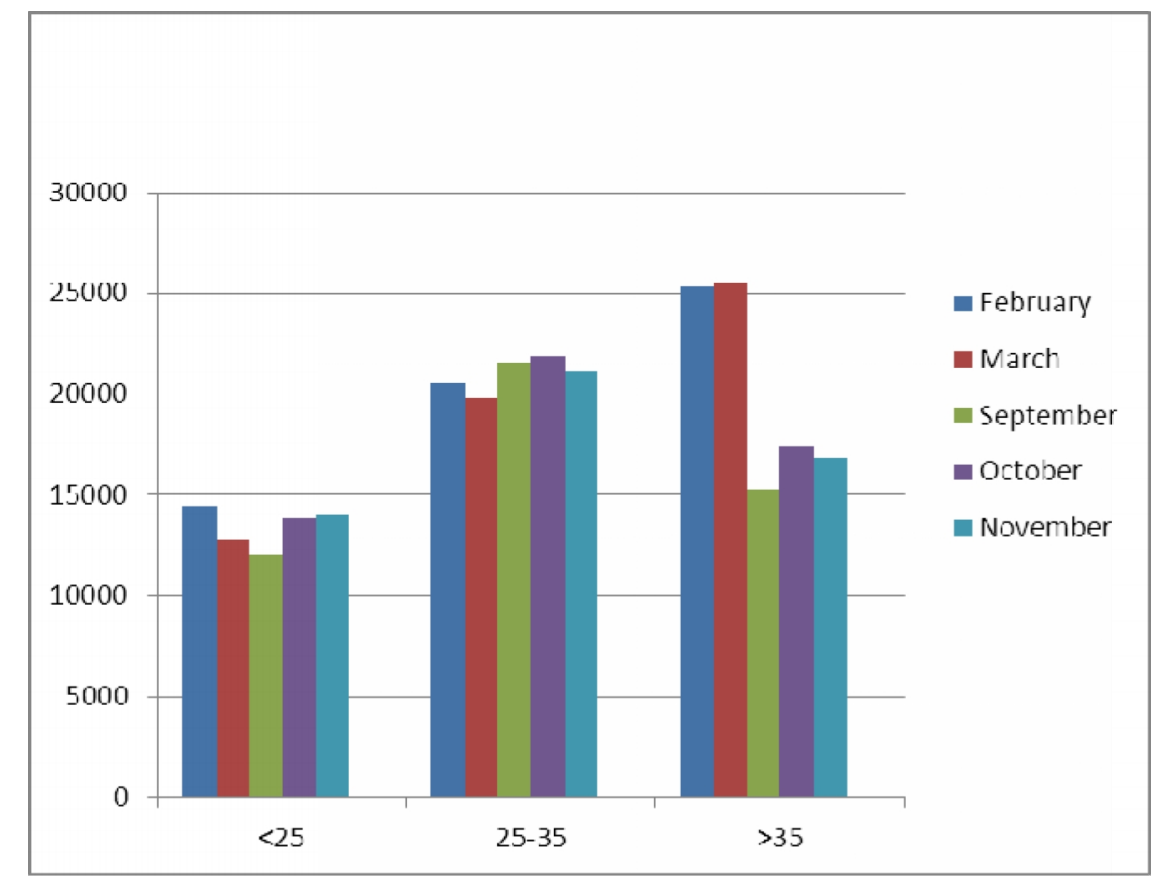

Fig. 1. Recombinant human erythropoietin (rhuEPO) utilization during the period of observation. baseline months (February, March) - I.V. administration; follow-up months (September, October, November) - S.C. administration

$Y$-axis: rhuEPO, Units/week; X-axis: rhuEPO use, grouped according to BMI $\left(\mathrm{kg} / \mathrm{m}^{2}\right)$

In keeping with overall burden of obesity in the local community [14,15], we found a significant burden of obesity in the $3^{\text {rd }}$ BMl category group, in those reaching or exceeding Class 2 obesity $\left(B M I>35 \mathrm{~kg} / \mathrm{m}^{2}\right.$ ). Among these subjects, we demonstrated a possible differential effect of obesity during IV to SC rhuEPO conversion. Compared with the US cohort of the Dialysis Outcomes and Practice Patterns Study (DOPPS), our BMI I and 2 categories sub-cohort had slightly lesser, while BMI category 3 had more excessive rhuEPO utilization, than reported from DOPPS (17,360 U/week) [16]. In our cohort, only the obese subjects showed the expected reduction in rhuEPO dose, suggesting these participants may account for a disproportionate amount of rhuEPO dose reduction during IV to SC 
conversion. While these changes take effect only in the markedly obese subjects remains unclear at present time. It may be more difficult to ensure truly subcutaneous administration in leaner individuals, the concern being that these individuals are actually receiving intramuscular injections a fair percentage of the time. Skeletal muscles are highly vascular organs and drugs are taken up rapidly by direct diffusion through the capillary walls. Thus, the most likely explanation is that in obese individuals, SC rhuEPO may have a different pharmacokinetics allowing for slower absorption and larger "area under the curve" exposure, whereas in overweight or normal weight subjects it may behave closer to intramuscular (IM) route. It is also interesting that in animal models rhuEPO has been shown, in dosage range above used to correct anemia of renal failure, to influence body composition and insulin sensitivity $[17,18]$.

Since rhuEPO's initial introduction [19], the benefits on quality of life certainly has been well recognized $[20,21]$ and it has been part of the standard armamentarium of nephrology providers. At present time, professional guidelines remain uncommitted on IV vs. SC route for in-center dialyzed patients [2]. On the other hand, the convenience and reduced discomfort of IV route is well established [4,22]. An additional driving force for IV route in the past (before January of 2011) was the reimbursement scheme of CMS offering separate payment for injectable medications received during renal dialysis [23]. Published studies to date are in some disagreement with regard to efficacy of conversion to reduce rhuEPO utilization. For example, in the VA Cooperative Study, SC (rather than IV) group assignment was associated with an approximately one-third dose requirement reduction (95 versus 140 U/kg per week) [4]. On the other hand, in the DOPPS study overall a $14 \%$ reduction was noted in association with SC route, but in the US cohort this reduction was only $3 \%$ [16]. Additionally, the lack of beneficial effect of SC conversion may also be attributed to the relatively good iron-supplied state of our cohort [24].

Other limitations of our study also should be strongly emphasized, including the singlecenter design, small number of participants, limited number of variables, as well as the lack of control group and loss during subsequent follow-up. Beyond iron saturation and ferritin level we did not measure other parameters of iron supply. Blood pressure results were not collected during the study and it is uncertain, whether IV to SC rhuEPO conversion influenced blood pressure control to any degree. We did not formally queried complaints of local discomfort with SC injection; however, the excellent acceptance rate of the conversion process ( 86 of 90 subjects, or $95.5 \%$ ) suggested this was not a major issue in our cohort. Furthermore, the lack of reaching nominal statistical significance should caution us from over-interpreting our results and should call on additional studies on the effect of obesity modifying efficacy of recombinant erythropoietin during IV vs. SC administration. Nonetheless, our main intention was to demonstrate in a pilot study what may be a generic concern with biological preparations and laying the groundwork for additional work in larger databases or prospective studies.

In summary, we found that SC conversion well accommodated in almost all subjects and SC route was not inferior to intravenous route during rhuEPO administration. Our results suggest that any possible dose reduction achieved via SC conversion of rhuEPO would be, if any, limited to subjects with Class 2 obesity (Body Mass Index $>35 \mathrm{~m} / \mathrm{kg}^{2}$ ). Nonetheless, while the rhuEPO reduction did not reach statistical significance $(p=0.081)$, the overall, unitlevel dose reduction of rhuEPO use in Class 2 obese was relatively large $(\sim 1 / 3)$, resulting in large potential cost-saving for the dialysis unit on the whole. This potential benefit is to be counter-balanced by patients' preference and elimination of discomfort of SC injections, when injecting IV rhuEPO directly into blood circuit directly during dialysis. 


\section{CONCLUSION}

Our results affirmed a strong statistical trend, but no conclusive proof that IV to SC conversion of rhuEPO administration may be differentially influenced by the presence of obesity. This finding may be relevant to other injectable hormones and cytokines of similar molecular size.

\section{CONSENT AND ETHICAL APPROVAL}

All authors hereby declare that this study have been examined and approved by the appropriate ethics committee of the University of Mississippi Human Research Office (formerly: Institutional Review Board) and approved in accordance with the ethical standards laid down in the 1964 Declaration of Helsinki, further revised in 1983 (IRB approval number \#2010-0028). The University of Mississippi Human Research Office has determined that individual consents from the study participants were not necessary.

\section{ACKNOWLEDGEMENT}

Acknowledgements Dr Michael Shoemaker-Moyle is currently affiliated with the Hampton Veterans Administration Medical Center and the Eastern Virginia Medical School, Hampton, VA. Dr. Lajos Zsom is currently affiliated with Principal SMO Kft, Budapest, Hungary. Dr. Mihály Tapolyai is currently affiliated with WJB Dorn VA Medical Center, Columbia, SC. We appreciated the assistance of Manjunath Ramaiah, M.D., during the data collection. Parts of this study were presented in poster format at the National Kidney foundation 2011 Spring Clinical Meeting, Las Vegas, NV.

\section{COMPETING INTERESTS}

The authors have no competing conflict of interests to report. The authors have no funding from any source.

\section{REFERENCES}

1. IV. NKF-K/DOQI Clinical Practice Guidelines for Anemia of Chronic Kidney Disease: update 2000. Administration of epoetin. Am J Kidney Dis. 2001;37(1 Suppl 1):S182238.

2. NKF-DOQI Clinical Practice Guidelines for Anemia of Chronic Renal Failure. Am J Kidney Dis. 2006;47((Suppl 4):S1.).

3. McFarlane PA, Hillmer MP, Dacouris N. A change from subcutaneous to intravenous erythropoietin increases the cost of anemia therapy. Nephron Clin Prac. 2007;107(3):c90-c96.

4. Kaufman JS, Reda DJ, Fye CL, et al. Subcutaneous compared with intravenous epoetin in patients receiving hemodialysis. Department of Veterans Affairs Cooperative Study Group on Erythropoietin in Hemodialysis Patients. N Engl J Med. 1998;339(9):578-583.

5. Raymond C, Collins DM, Bernstein KN, Skwarchuk DE, Vercaigne LM. Erythropoietinalpha dosage requirements in a provincial hemodialysis population: effect of switching from subcutaneous to intravenous administration. Nephron Clin Prac. 2005;102(34):c88-c92. 
6. Steinkampf MP, Hammond KR, Nichols JE, Slayden SH. Effect of obesity on recombinant follicle - stimulating hormone absorption : subcutaneous versus intramuscular administration. Fertil Steril. 2003;80(1):99-102.

7. Chan $\mathrm{CC}, \mathrm{Ng} \mathrm{EH}$, Chan $\mathrm{MM}$, et al. Bioavailability of hCG after intramuscular or subcutaneous injection in obese and non - obese women. Hum Reprod. 2003;18(11):2294-2297.

8. Besarab A, Reyes CM, Hornberger J. Meta - analysis of subcutaneous versus intravenous epoetin in maintenance treatment of anemia in hemodialysis patients. Am J Kidney Dis. 2002;40(3):439-446.

9. Smith KJ, Bleyer AJ, Little WC, Sane DC. The cardiovascular effects of erythropoietin. Cardiovasc Res. 2003;59(3):538-548.

10. Besarab A, Bolton WK, Browne JK, et al. The effects of normal as compared with low hematocrit values in patients with cardiac disease who are receiving hemodialysis and epoetin. N Eng J Med. 1998;339(9):584-590.

11. Pfeffer MA, Burdmann EA, Chen CY, et al. A trial of darbepoetin alfa in type 2 diabetes and chronic kidney disease. N Eng J Med. 2009;361(21):2019-2032.

12. Singh AK, Szczech L, Tang KL, et al. Correction of anemia with epoetin alfa in chronic kidney disease. N Eng J Med. 2006;355(20):2085-2098.

13. Palmer SC, Navaneethan SD, Craig JC, et al. Meta-analysis: erythropoiesisstimulating agents in patients with chronic kidney disease. Ann Intern Med. 2010;153(1):23-33.

14. Flessner MF, Wyatt SB, Akylbekova EL, et al. Prevalence and awareness of CKD among African Americans: the Jackson Heart Study. Am J Kidney Dis. 2009;53(2):238-247.

15. Fülöp T, Hickson DMA, Wyatt SB, et al. Sleep-disordered breathing symptoms among African-Americans in the Jackson Heart Study. Sleep Med. 2012;13(8):1039-1049.

16. Pisoni RL, Bragg-Gresham JL, Young EW, et al. Anemia management and outcomes from 12 countries in the Dialysis Outcomes and Practice Patterns Study (DOPPS). Am J Kidney Dis. 2004;44(1):94-111.

17. Foskett A, Alnaeeli M, Wang L, Teng R, Noguchi CT. The effects of erythropoietin dose titration during high-fat diet-induced obesity. J Biomed Biotechnol. 2011;2011.

18. Teng R, Gavrilova O, Suzuki N, et al. Disrupted erythropoietin signalling promotes obesity and alters hypothalamus proopiomelanocortin production. Nature Comm. 2011;2:520.

19. Eschbach JW, Egrie JC, Downing MR, Browne JK, Adamson JW. Correction of the anemia of end-stage renal disease with recombinant human erythropoietin. $\mathrm{N}$ Eng $\mathrm{J}$ Med. 1987;316(2):73-78.

20. Evans RW, Rader B, Manninen DL. The quality of life of hemodialysis recipients treated with recombinant human erythropoietin. JAMA. 1990;263(6):825-830.

21. Kimmel PL, Cohen SD, Weisbord SD. Quality of life in patients with end-stage renal disease treated with hemodialysis: survival is not enough! J Nephrol. 2008;21:S54.

22. Kinney R. Annual Report: ESRD Clinical Performance Measures Project. Am J Kidney Dis. 2006;48:S1-S105.

23. Thamer M, Zhang Y, Kaufman J, Stefanik K, Cotter DJ. Factors influencing route of administration for epoetin treatment among hemodialysis patients in the United States. Am J Kidney Dis. 2006;48(1):77-87. 
24. Pizzarelli F, David S, Sala P, Icardi A, Casani A. Iron-replete hemodialysis patients do not require higher EPO dosages when converting from subcutaneous to intravenous administration: Results of theitalian study on erythropoietin converting (ISEC). Am J Kidney Dis. 2006;47(6):1027-1035.

(C) 2014 Csongrádi et al.; This is an Open Access article distributed under the terms of the Creative Commons Attribution License (http://creativecommons.org/licenses/by/3.0), which permits unrestricted use, distribution, and reproduction in any medium, provided the original work is properly cited.

Peer-review history:

The peer review history for this paper can be accessed here: http://www.sciencedomain.org/review-history. php?iid=215\&id=12\&aid=2011 